\title{
Call for Special Issue Papers: Neurotrauma Reports Null Hypothesis
}

\section{Deadline for Manuscript Submission: Manuscripts submitted will be considered on a rolling basis.}

\author{
Guest Editors: Dr. Andreas Jeromin, Chief Scientific Officer, Cohen Veterans' Bioscience \\ and Dr. Sandra Petty, Chief Executive Officer, Center for Biomedical Research Transparency
}

Neurotrauma Reports Null Hypothesis is a special section reserved for the publication of well-conducted negative, inconclusive, and replicative studies. The goal of this section is to catalyze a culture shift in science whereby publication of such studies becomes standard practice. This collection will publish on a rolling basis in Neurotrauma Reports, a fully online open access journal.

Our scientific culture has an embedded ethos that publishing positive results equates to more success, productivity, interest and value. This has become apparent as the proportion of positive results published in scientific literature has increased by $6 \%$ per year since $1990^{1}$ and, in neuroscience, estimates indicate that approximately $85 \%$ of manuscripts published include only positive results ${ }^{2}$. Yet publishing well-conducted negative studies and replicating previously published studies leads to transparent and well-balanced reporting, tenets that are central to rigor-

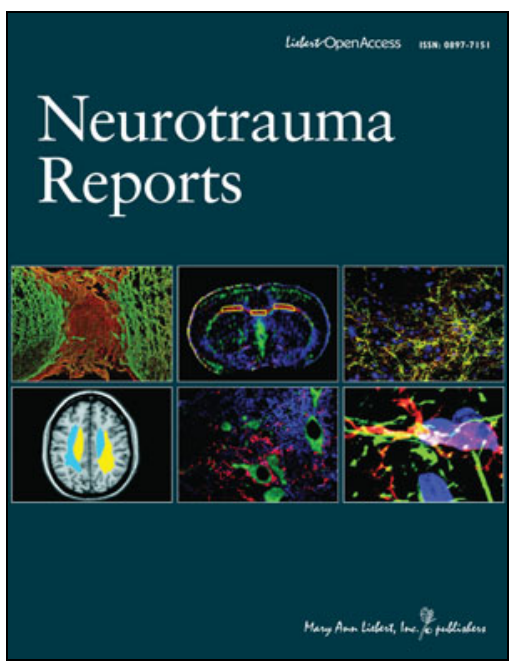
ous and efficient experimental design, scientific advancement and improving patient outcomes.

Neurotrauma Reports Null Hypothesis is a collaborative initiative with the Center for Biomedical Research Transparency and Cohen Veterans' Bioscience. The Null Hypothesis model has already demonstrated its potential to promote balance in academic publications by urging more researchers in the neurology and cardiology fields to submit important negative and inconclusive research findings for peer review. It is our hope that with this special section, we will engage the broader scientific community to ensure best practices are adopted and that by including all well-conducted studies, scientific research will progress faster, leading to a better understanding of the biological underpinnings of brain injury related disorders, and the development of personalized therapeutic approaches.

Neurotrauma Reports Null Hypothesis is open to well-conducted negative, inconclusive and replicative studies within the scope of Neurotrauma Reports, including but not limited to traumatic brain injury, stroke and spinal cord injury. Studies should be full length empirical studies, adhere to applicable research rigor standards, 
include an appropriate description of the methodology, and clearly describe statistical analyses and approaches used to support conclusions. Researchers who submit studies for publication in this special collection may also include extended supplementary materials.

All manuscripts should be submitted online using the ScholarOne submission site. Please submit under the Null Hypothesis manuscript category. All submissions will be subject to a rigorous peer review. We encourage submissions of original research articles, reviews, and perspectives.

CVB and CBMRT are committed to promoting open access. In instances where authors' research funding arrangements do not cover APCs, these costs will be eligible for coverage as part of the Null Hypothesis initiative. Please contact sarah@cbmrt.org for further information.

Visit Neurotrauma Reports to learn more, read past issues, and view author submission guidelines. Queries to the editor to propose a topic prior to submission are encouraged. Please contact Jean Ann Sanford (j.neurotrauma@gmail.com) to initiate your query or for any further details.

\section{Visit the Instructions for Authors: www.liebertpub.com/neur}

\section{Submit your paper for peer review online: https://mc.manuscriptcentral.com/neur}

